\title{
Is the exhaled breath temperature in lung cancer influenced by airways neoangiogenesis or by inflammation?
}

\author{
Giovanna E. Carpagnano ${ }^{1} \cdot$ Donato Lacedonia $^{1} \cdot$ Antonio Spanevello $^{2,3} \cdot$ \\ Grazia Cotugno $^{1} \cdot$ Valerio Saliani $^{1} \cdot$ Domenico Martinelli $^{4} \cdot$ Maria P. Foschino-Barbaro $^{1}$
}

Received: 27 July 2015/Accepted: 17 August 2015/Published online: 1 September 2015

(C) Springer Science+Business Media New York 2015

\begin{abstract}
Recently the exhaled breath temperature (EBT) was seen to increase in non-small cell lung cancer and was subsequently proposed as a possible non-invasive tool for its diagnosis. The need for further studies that confirm the previous findings and support the potential scope of this method underlies the main aim of this study that seeks to explore the pathogenic mechanisms determining the EBT in lung cancer. We enrolled 44 consecutive patients with a radiological suspicion of lung cancer and ten healthy nonsmoker volunteers, after which their EBT was measured. On the same day, the subjects underwent breath condensate collection for the measurement of leukotriene (LTB)-4 and of the vascular endothelial growth factor (VEGF), the former being a marker of airways inflammation and the latter of neoangiogenesis. We confirmed the presence of a higher EBT in lung cancer patients compared to the controls. The multiple linear regression model showed that the exhaled VEGF was the only predictor of elevations of EBT. In conclusion, it can be stated that for the first time in this study, we have shown that EBT is higher in subjects with lung cancer and that the airways angiogenesis drives the increase in EBT in lung cancer. Moreover, the study suggests the potential for the use of EBT in monitoring the
\end{abstract}

Giovanna E. Carpagnano

giovannaelisiana.carpagnano@unifg.it

1 Department of Medical and Surgical Sciences, Institute of Respiratory Disease, University of Foggia, Foggia, Italy

2 Institute of Respiratory Disease, University of Insubria, Varese, Italy

3 Fondazione Salvatore Maugeri, IRCCS, Tradate, Italy

4 Section of Hygiene, Department of Medical Sciences, University of Foggia, Foggia, Italy lung cancer progression, although the implementation of more in-depth studies to verify this result is recommended.

Keywords Lung cancer - Non-invasive methods . Angiogenesis - Airways inflammation - Exhaled breath condensate $\cdot$ Exhaled breath temperature

\section{Introduction}

Cancer entails an abnormal growth of cells which tend to proliferate in an uncontrolled way and, in some cases, end up metastasizing [1]. Inflammation has been recently recognized as an important pathogenetic trigger in cell cancer transformation, promoting an environment that is conducive to carcinogenesis [2]. Currently there is a large amount of clinical data confirming the inflammatory origin of lung cancer [2-4]. Indeed, the formation of the new blood vessels controlled by certain chemicals, which when stimulated repair damaged cells or form new ones, is an essential condition for cancer evolution and diffusion [5, 6]. A recent intriguing line of research (that is also a prerequisite for any strategy to improve the lung cancer outcome) focused on finding new early markers of lung cancer using non-invasive methods of sampling. Among those, the exhaled breath condensate (EBC) and the exhaled breath temperature (EBT) are recently receiving a lot of interest for their suitability in this category of patients who are often frustrated by the numerous diagnostic tests they underwent and therefore for their easy acceptability. Several studies today exist on EBC [2-4, 7-9] in lung cancer, while there are fewer on EBT, a new validated marker of airways inflammation that is broadly applied to the study of asthma. In recent years, the measurement of EBT has been proposed in the area of revealing neoplastic alterations 
[10]. In this regard, our group recently measured, for the first time to our knowledge, the EBT in a group of nonsmall cell lung cancer (NSCLC) patients and controls, and reported an increase in EBT in oncologic patients [11]. Furthermore, some study groups have made a significant contribution in supporting the usefulness of the EBC in the attempt to promote interest in the early diagnosis of lung cancer [3, 4, 8, 12-16]. Although the exhaled breath condensate is not able to give information about the cytologic profile of airways, it appears to be useful in offering feedback on the oncogenesis through the dosage of soluble and genetic tumour markers in airways [2-4, 9, 13, 15]. In a previous study, we also confirmed the specificity of the EBC, reporting the same information carried by lung cancer tissue [13].

In this study, we aim to focus on the pathogenetic mechanisms involved in the increase in the EBT as we believe that it has the scope to become an important early non-invasive diagnostic tool.

In consideration that the temperature signal reflects important pathogenetic moments of lung cancerogenesis such as inflammation and hypervascularization of airways, we now want to explore possible correlations between the EBT in lung cancer patients and two markers, i.e. inflammation and neoangiogenetic markers, meaning leukotriene (LTB)-4 and the vascular endothelial growth factor (VEGF). Both these markers have already been found to be dosable in the EBC and at increased levels in lung cancer patients [2, 16].

\section{Materials and methods}

During a 12-month period from January 2012 to January 2013, 44 consecutive patients (opportunistic sample) with a radiological suspicion of lung cancer and ten healthy nonsmoker volunteers who agreed to take part in the study were enrolled at the Unit of Thoracic Surgery, Casa di Cura La Madonnina, Bari, and at the Section of Respiratory Disease, University of Foggia (Table 1). Written informed consent was obtained from all subjects upon approval of the study by the ethic committees of the two hospitals.

All patients were enrolled in the study immediately before histological diagnosis. Furthermore, none of them had received any form of anti-cancer therapy or undergone either any invasive diagnostic procedure or any primary lung surgery. Following cytohistological diagnosis, the patients with cancer underwent standard staging procedures consisting of a physical examination, serum chemistry analysis, brain, chest and abdomen CT scans, radionuclide bone scan and bronchoscopy. The diagnosis of NSCLC was done either by bronchoscopic biopsy or by transthoracic needle aspiration.
All of the patients enrolled underwent EBT collection and the collection of the exhaled breath condensate during the morning at enrolment, starting from 9 a.m. Ear temperature was measured by means of an ear thermometer (OMRON, Model Gentle Temp 5100, Healthcare Europe, the Netherlands) to exclude that patients had a fever.

The diagnosis of lung cancer was performed in 26 patients, while others resulted in false positive.

Squamous cell carcinoma was diagnosed in 12 subjects, whereas the remaining lung cancer patients received a cytohistological diagnosis of adenocarcinoma. Overall, NSCLC patients were classified as stage I in 4 cases, stage II in 3 cases, stage III in 10 cases and stage IV in 9 cases.

Thirteen of the lung cancer patients were current smokers (54 \pm 6.3 pack/year); seven were ex-smokers (45 \pm 4 pack/year) and had quit at least 3 years before; and six were non-smokers. Twelve of the false-positive patients were current smokers (50 \pm 5.1 pack/year); five were ex-smokers ( $45 \pm 4$ pack/year) and had quit at least 3 years before; and one was a non-smoker. The healthy volunteers were all non-smokers. Thirteen NSCLC patients and 12 false-positive patients were COPD.

\section{Exhaled breath temperature measurement}

EBT was measured with an X-Halo device (Delmedica Investments, Singapore) according to previously validated methods $[15,16]$. Briefly, patients were requested to inhale freely through the nose and to exhale into the device at a rate and depth typical of their normal tidal breathing rhythm. The procedure was continued until the built-in software of the instrument indicated that the measured value was stable, fulfilling the criteria of a previously described mathematical model; the instrument processed an incremental temperature curve in relation to the initial temperature of the air in the thermal chamber of the instrument, and was able to capture the achievement of the temperature plateau with an error $<2 \%$ [15]. The reproducibility of the EBT measurements performed by X-Halo had been previously demonstrated to have an intraclass correlation coefficient of 0.99 [16]. The test was carried out at a room temperature of $19-25^{\circ} \mathrm{C}$ and at a relative humidity of $30-60 \%$.

\section{Exhaled breath condensate}

The oral exhaled breath condensate was collected using a condenser which allowed for the non-invasive collection of the non-gaseous components of the expired air (EcoScreen, Jaeger, Wurzburg, Germany). Subjects breathed through a mouthpiece and a two-way non-rebreathing valve, which also served as a saliva trap. They were asked to breathe at a normal frequency and at tidal volume, wearing a nose clip, 
Table 1 NSCLC patients, false positive and healthy controls' description by sex, age, smoke habit, COPD, FEV1 \%, FVC \%, $\mathrm{PaO} 2, \mathrm{PaCO} 2$

\begin{tabular}{llll}
\hline & Cases $(N=26)$ & False positive $(N=18)$ & Healthy controls $(N=10)$ \\
\hline Sex & & & 5 \\
$\quad$ Male & 19 & 11 & $57.1 \pm 8.4$ \\
Age (years) & $62.7 \pm 10.1$ & $61.3 \pm 13.0$ & \\
Smoke habit & & 1 & 10 \\
$\quad$ Never smoked & 5 & 12 & - \\
Smoker & 13 & 5 & - \\
Ex-smoker & 6 & $52.4 \pm 5.1$ & \\
Pack/year $($ mean \pm SD) & $54 \pm 6.3$ & & 0 \\
COPD & & 12 & $98.3 \pm 8.1$ \\
Yes & 13 & $58.5 \pm 10.3$ & $100.1 \pm 7.5$ \\
FEV1 \% (mean \pm SD) & $60.5 \pm 12.1$ & $67.21 \pm 10.0$ & $97.2 \pm 1.8$ \\
FVC \% (mean \pm SD) & $65.1 \pm 11.5$ & $71.9 \pm 10.3$ & $39.5 \pm 1.7$ \\
PaO2 (mean \pm SD) & $72.48 \pm 11.7$ & $37.3 \pm 3.5$ & \\
PaCO2 (mean \pm SD) & $39.5 \pm 4.2$ & & \\
\hline
\end{tabular}

for a period of $10 \mathrm{~min}$. Subjects were instructed to swallow any saliva they felt in their mouth. The condensate, when amounting to at least $1 \mathrm{ml}$, was collected as ice at $-20^{\circ} \mathrm{C}$, transferred to Eppendorf tubes and immediately stored at $-80{ }^{\circ} \mathrm{C}$. Samples were stored for not more than 2 months before analysis. Amylase concentration was measured in all samples to exclude any salivary contamination.

\section{Measurement of LTB4}

A specific enzyme immunoassay (Human VEGF, Thermo Scientific) was used to measure LTB4 in breath condensate. The intraassay and interassay variability were $<10 \%$. The specificity was $100 \%$, and the detection limit of the assay was $5 \mathrm{pg} / \mathrm{ml}$. The reproducibility of the repeated measurements of exhale LTB-4 was confirmed by the Bland and Altman test and by the variation coefficient [17].

\section{Measurement of VEGF}

A specific enzyme immunoassay (EIA) kit (Human VEGF, Thermo Scientific) was used to measure VEGF concentrations in breath condensate. The intraassay and interassay variability were $<10$ and $<10 \%$. The detection limit of the assays was $19.4 \mathrm{pg} / \mathrm{ml}$. The reproducibility of the repeated measurements of exhaled VEGF was confirmed by the Bland and Altman test and by the variation coefficient [17].

\section{Statistics}

To assess significant differences between cases and controls, we used the nonparametric Kruskal-Wallis test and Chi-square test for continuous variables (age, number of packs/year, FEV1 \%, FVC \%, PaO2, PaCO2) and for categorical variables (sex, smoke habits, COPD), respectively.
The differences between cases and controls or among the group of cases (histological diagnosis and stage) were assessed by the Mann-Whitney test. Temperature was correlated with number of packs/year, exhaled LTB-4 and exhaled VEGF using Spearman's rank correlation test. To assess the effect of the variables LTB4, VEGF, pack/year and COPD on the EBT we constructed a multiple linear regression model.

We considered a $p$ value of $<0.05$ to be significant. Data were analysed using STATA PM 10.1 for MAC OS 10.6.

\section{Results}

The characteristics of the three groups are described in Table 1. In this study, we found higher EBT in NSCLC patients compared to the false-positive patients and to the healthy controls $\left(35.5 \pm 0.72{ }^{\circ} \mathrm{C}\right.$ vs $33.3 \pm 0.40$ and $31.8 \pm 0.32{ }^{\circ} \mathrm{C}$ ) and a correlation between EBT and number of packs/year $(r=0.80, p<0.001)$. In the NSCLC cases, we found no differences in exhaled temperature for hystological type (squamous carcinoma vs adenocarcinoma), while higher exhaled temperatures were observed in more advanced stages (III-IV; $\chi^{2}=7.697$, $p=0.005)$.

Higher concentrations of LTB-4 and VEGF were found in the exhaled breath condensate of NSCLC patients than in false-positive patients and controls (65.5 \pm 0.2 vs $58.8 \pm 1.1$ and $40.1 \pm 0.8 \mathrm{pg} / \mathrm{ml} ; \quad 24.8 \pm 0.6 \quad$ vs $18.9 \pm 0.7$ and $9.1 \pm 0.6 \mathrm{pg} / \mathrm{ml}$ ). A positive correlation was observed between EBT, exhaled LTB4 and VEGF ( $r=0.7, p<0.001 ; r=0.7, p<0.001)$.

When we tested the relative contribution of exhaled LTB-4 and exhaled VEGF on EBT using multistep regression models, only exhaled VEGF was found to be 
correlated with EBT (coef $0.13,95 \%$ CI $0.03-0.23$, $p=0.02$; Table 2).

\section{Discussion}

In this study for the first time, we showed that EBT is higher in subjects with lung cancer and that the airways angiogenesis drives the increase in EBT in lung cancer. Moreover, the study suggests the potential for the use of EBT in monitoring lung cancer progression, although, as stated above, further in-depth studies to verify this result are recommended.

However, when we tested the relative contribution of exhaled LTB-4 and exhaled VEGF on EBT, the multistep regression models showed that only the exhaled VEGF was found to be correlated with EBT.

An increasing interest in non-invasive methods to study airways inflammation is now being directed to the measurement of the EBT. In the last decade, the set-up of a third-generation device for the measurement of the EBT, the xhalo, has increased the number of studies on this marker in several inflammatory respiratory diseases and particularly in asthma $[15,16]$.

By starting from the recent advances in cancer biology that both point to a role of inflammatory signalling in lung cancer and encourage the reconsideration of the diagnostic and prognostic value of inflammatory markers [2], we recently measured the EBT in a group of NSCLC patients [11]. Our preliminary results were interesting as they showed a significantly higher EBT in lung cancer patients and a strong association of EBT with the number of cigarettes smoked [11]. From the analysis of the ROC curve, we furthermore identified a cut-off value for the EBT over which $92.9 \%$ of the subjects were diagnosed as having lung cancer; this led us to infer that there was potential for using the EBT in the early diagnosis of this disease [11]. In this previous study, we supposed that in addition to the airways inflammation, the hypervascularization by new vessels (that characterize the evolution of lung cancer) could also be responsible for the observed increase in EBT.

Table 2 Multiple linear regression model

\begin{tabular}{lrrrrr}
\hline EBT & Coef. & \multicolumn{1}{l}{$t$} & $p$ & $95 \%$ CI & \\
\hline LTB4 & 0.11 & 1.67 & 0.11 & -0.02 & 0.24 \\
VEGF & 0.13 & 2.56 & 0.02 & 0.03 & 0.23 \\
P/Y & 0.00 & -0.30 & 0.77 & -0.02 & 0.01 \\
COPD & 0.51 & 1.46 & 0.16 & -0.21 & 1.23 \\
Cons & 24.93 & 7.08 & 0.00 & 17.72 & 32.14 \\
\hline
\end{tabular}

In the light of the strategic potential of using EBT in oncology, we set out to use this study to verify the involvement of each of these pathogenetic key mechanisms in determining lung cancer via an analysis of the increase in EBT. Staying in the subject area of non-invasive samples, we selected the EBC in order to analyse the airways of our lung cancer patients, measuring (in that sample) two markers already found to have increased in the EBC of NSCLC patients, namely LTB-4 and VEGF [2, 16, 18-23]. Having selected the first marker, the LTB-4 was recognized to play a crucial role in neutrophil recruitment into airways during lung cancer and was found to be significantly higher as the cancer stages progressed $[2,18,19]$ and also to be correlated with smoking. The angiogenic marker that we analysed, the VEGF, was also reported to be higher in advanced T-stage [20] and was recently proposed as an early diagnostic and prognostic marker for lung cancer [16, 19, 20, 24].

In this study, we not only confirmed the previously reported correlation between EBT and packs/year but also found a new positive correlation with both exhaled LTB-4 and exhaled VEGF. However, when we applied the multistep regression models to exhaled LTB-4 and exhaled VEGF, only the latter resulted as being correlated with the EBT. This finding has given an important contribution towards solving the main point of this study as it highlights the key role of angiogenesis in increasing the EBT. The elevation of the EBT observed in more advanced stages of lung cancer is therefore the expression of an increased neoangiogenesis that drives the metastatic diffusion of the disease. Our results have led us to infer the potential for using the EBT in monitoring lung cancer progression.

That said, this study could be criticized both for using the EBT and for analysing the soluble markers in the EBC that are not employed in clinical practice yet. Nevertheless, our group strongly support these methods and believe that they have a great potential (non-invasiveness and repeatability of the technique) in both clinical and research settings. Furthermore, we tried to solve the high variability and needs standardization of these methods by always performing the analysis in the same laboratory and involving the same technician in the collection and blind analysis of the samples.

In conclusion, although this first-time study investigated pathogenetic mechanisms on the basis of the EBT in lung cancer, giving a possible interpretation of the observed increased EBT as a result of neoangiogenesis, our results need to be followed up by a validation in a larger population and our supposition confirmed with an in-depth exploration of the involvement of both airways inflammation and neoangiogenesis in driving EBT, testing other validated markers. 


\section{Compliance with ethical standards}

Conflict of interest The authors declare that they have no conflict of interest.

\section{References}

1. http://www.medicinenet.co.

2. Carpagnano GE, Palladino GP, Lacedonia D, Koutelou A, Orlando S, Foschino-Barbaro MP. Neutrophilic airways inflammation in lung cancer: the role of exhaled LTB-4 and IL-8. BMC Cancer. 2011;7(11):226.

3. Carpagnano GE, Spanevello A, Curci C, Salerno F, Palladino GP, Resta O, Di Gioia G, Carpagnano F, Foschino Barbaro MP. IL-2, TNF-alpha, and leptin: local versus systemic concentrations in NSCLC patients. Oncol Res. 2007;16(8):375-81.

4. Carpagnano GE, Resta O, Foschino-Barbaro MP, Gramiccioni E, Carpagnano F. Interleukin-6 is increased in breath condensate of patients with non-small cell lung cancer. Int J Biol Markers. 2002;17(2):141-5.

5. Folkman J. What is the evidence that tumours are angiogenesis dependent? J Natl Cancer Inst. 1990;82:4-6.

6. Chatterjee S, Bhattacharjee B. Use of natural molecules as antiangiogenic inhibitors for vascular endothelial growth factor receptor. Bioinformation. 2012;8(25):1249-54.

7. Carpagnano GE, Kharitonov SA, Resta O, Foschino-Barbaro MP, Gramiccioni E, Barnes PJ. Increased 8-isoprostane and interleukin-6 in breath condensate of obstructive sleep apnea patients. Chest. 2002;122(4):1162-7.

8. Carpagnano GE, Lacedonia D, Palladino GP, Koutelou A, Martinelli D, Orlando S, Foschino-Barbaro MP. Could exhaled ferritin and SOD be used as markers for lung cancer and prognosis prediction purposes? Eur J Clin Investig. 2012;42(5):478-86.

9. Carpagnano GE, Spanevello A, Carpagnano F, Palladino GP, Prato R, Martinelli D, Digioia G, Foschino-Barbaro MP. Prognostic value of exhaled microsatellite alterations at $3 p$ in NSCLC patients. Lung Cancer. 2009;64(3):334-40.

10. Stefanadis C, Chrysohoou C, Panagiotakos DB, Passalidou E, Katsi V, Polychronopoulos V, Toutouzas PK. Temperature differences are associated with malignancy on lung lesions: a clinical study. BMC Cancer. 2003;6(3):1.

11. Carpagnano GE, Lacedonia D, Spanevello A, Martinelli D, Saliani V, Ruggieri C, Foschino-Barbaro MP. Exhaled breath temperature in NSCLC: could be a new non-invasive marker? Med Oncol. 2014;31(5):952. doi:10.1007/s12032-014-0952-1.

12. Chan HP, Lewis C, Thomas PS. Exhaled breath analysis: novel approach for early detection of lung cancer. Lung Cancer. 2009;63:164-8.
13. Carpagnano GE, Foschino-Barbaro MP, Spanevello A, Resta O, Carpagnano F, Mulé G, Pinto R, Tommasi S, Paradiso A. 3p microsatellite signature in exhaled breath condensate and tumor tissue of patients with lung cancer. Am J Respir Crit Care Med. 2008;177(3):337-41.

14. Carpagnano GE, Foschino-Barbaro MP, Mulé G, Resta O, Tommasi S, Mangia A, Carpagnano F, Stea G, Susca A, Di Gioia $\mathrm{G}$, De Lena M, Paradiso A. 3p microsatellite alterations in exhaled breath condensate from patients with non-small cell lung cancer. Am J Respir Crit Care Med. 2005;172(6):738-44.

15. Gessner C, Kuhn H, Toepfer K, Hammerschmidt S, Schauer J, Wirtz H. Detection of p53 gene mutations in exhaled breath condensate of non-small cell lung cancer patients. Lung Cancer. 2004;43(2):215-22.

16. Gessner C, Rechner B, Hammerschmidt S, Kuhn H, Hoheisel G, Sack U, Ruschpler P, Wirtz H. Angiogenic markers in breath condensate identify non-small cell lung cancer. Lung Cancer. 2010;68:177-84.

17. Popov TA, Dunev S, Kralimarkova TZ, Kraeva S, DuBuske LM. Evaluation of a simple, potentially individual device for exhaled breath temperature measurement. Respir Med. 2007;101(10): 2044-50.

18. Xepapadaki P, Xatziioannou A, Chatzicharalambous M, Makrinioti H, Papadopoulos NG. Exhaled breath temperature increases during mild exacerbations in children with virus-induced asthma. Int Arch Allergy Immunol. 2010;153(1):70-4.

19. Bland JM, Altman DG. Measuring agreement in method comparison studies. Stat Method Med Res. 1999;8:135-60.

20. Antczak A, Piotrowski W, Marczak J, Ciebiada M, Gorski P, Barnes PJ. Correlation between eicosanoids in bronchoalveolar lavage fluid and in exhaled breath condensate. Dis Markers. 2011;30(5):213-20.

21. Ciebiada M, Górski P, Antczak A. Eicosanoids in exhaled breath condensate and bronchoalveolar lavage fluid of patients with primary lung cancer. Dis Markers. 2012;32(5):329-35.

22. Dalaveris E, Kerenidi T, Katsabeki-Katsafli A, Kiropoulos T, Tanou K, Gourgoulianis KI, Kostikas K. VEGF, TNF-alpha and 8 -isoprostane levels in exhaled breath condensate and serum of patients with lung cancer. Lung Cancer. 2009;64(2):219-25.

23. Urbaniak Agnieszka, Zięba Marek, Zwolińska Anna, Szkudlarek Urszula, Łuczyńska Maria, Noweta Krzysztof, Kwiatkowska Sylwia. Comparison of selected markers of local and systemic inflammation in patients with community-acquired pneumonia and pneumonia co-existing with lung cancer. Pneumonol Alergol Pol. 2011;79(2):90-8.

24. Jemal A, Thomas A, Murray T, Thun M. Cancer statistics, 2002. CA Cancer J Clin. 2002;52:23-47. 